\title{
RECONSTRUCTION OF THE HEMIPELVIS AFTER THE EXCISION OF MALIGNANT TUMOURS
}

\author{
COMPLICATIONS AND FUNCTIONAL OUTCOME OF PROSTHESES
}

ADESEGUN ABUDU, ROBERT J. GRIMER, STEPHEN R. CANNON, SIMON R. CARTER, RODNEY S. SNEATH

From the Royal Orthopaedic Hospital Oncology Service, Birmingham, England

and the Royal National Orthopaedic Hospital, Stanmore, England

We treated 35 patients with primary malignant tumours of the periacetabular area by resection and prosthetic reconstruction of the defect. At a mean follow-up of 84 months, 15 patients $(43 \%)$ were free from disease. The most common complications were deep infection (26\%), local recurrence $(24 \%)$ and recurrent dislocation of the hip $(17 \%)$. The surviving patients achieved an average of $70 \%$ of their premorbid function.

This method of reconstruction has a high morbidity and should be performed only at specialist centres, but the functional and oncological outcomes are satisfactory.

J Bone Joint Surg [Br] 1997;79-B:773-9.

Received 25 March 1996; Accepted after revision 12 May 1997

About $15 \%$ of all primary malignant bone tumours involve the pelvis. ${ }^{1}$ Advances in the control of systemic and local disease by effective chemotherapy have improved survival and allowed more limb-preserving surgery. The overall survival of patients after limb-salvage surgery or amputation is similar when adequate excision margins are achieved. $^{2-4}$ The anatomy and the usually large size of pelvic tumours make adequate excision with preservation

A. Abudu, FRCS, Orthopaedic Specialist Registrar

R. J. Grimer, FRCS, Consultant Orthopaedic Oncologis

S. R. Carter, FRCS, Consultant Orthopaedic Oncologist

R. S. Sneath, FRCS, Former Director and Consultant Orthopaedic Oncologist

Royal Orthopaedic Hospital Oncology Service, Bristol Road North, Northfield, Birmingham B31 2AP, UK.

S. R. Cannon, FRCS, Consultant Orthopaedic Oncologist

Royal National Orthopaedic Hospital, Brockley Hill, Stanmore, Middlesex HA7 4LP, UK.

Correspondence should be sent to Mr R. J. Grimer.

(c)1997 British Editorial Society of Bone and Joint Surgery 0301-620X/97/56749\$2.00 of the limb a daunting challenge. Limb salvage for malignant pelvic tumours can preserve more function and avoid some of the social and psychological costs associated with hindquarter amputation.

Early surgical attempts to excise pelvic tumours were made by Enneking ${ }^{5}$ and by Steel, ${ }^{6}$ and were associated with high morbidity and mortality. Recent developments in imaging, anaesthesia, blood transfusion and surgery have stimulated development of improved techniques and enlarged the indications for limb preservation in patients with malignant pelvic bone tumours.

Adequate excision of malignant periacetabular tumours often requires complete excision of the acetabulum and partial or complete excision of the pubis and ischium as Enneking and Dunham ${ }^{7}$ type-II or type II-III pelvic excisions (Fig. 1). Extra-articular excision of the hip within its enveloping capsule may be needed, leaving a flail hip or pseudarthrosis, but arthrodesis of the femur to the remaining ilium, pubis, ischium or sacrum can provide improved stability. ${ }^{7}$ Flail hip, pseudarthrosis and arthrodesis all leave considerable limb shortening, poor function, pelvic tilt and impaired gait, and may lead to back pain. ${ }^{8}$

The emphasis is now on anatomical reconstruction by biological or mechanical means. Biological reconstruction uses allografts ${ }^{9}$ or reimplantation of excised bone after sterilisation by autoclaving. ${ }^{10,11}$ Allografts may be combined with a prosthesis as a composite. ${ }^{12}$ We have used mechanical reconstruction of the defect with custom-made prosthetic implants over the last 25 years. These provide maintenance of limb length and early restoration of function.

We now evaluate our experience, the surgical and technical complications, and the functional outcome of prosthetic reconstruction of the hemipelvis and hip after the excision of malignant periacetabular tumours.

\section{PATIENTS AND METHODS}

Between 1971 and 1994, a total of 35 patients had excision of periacetabular tumours for malignant bone tumours at either the London or the Birmingham Bone Tumour Treat- 

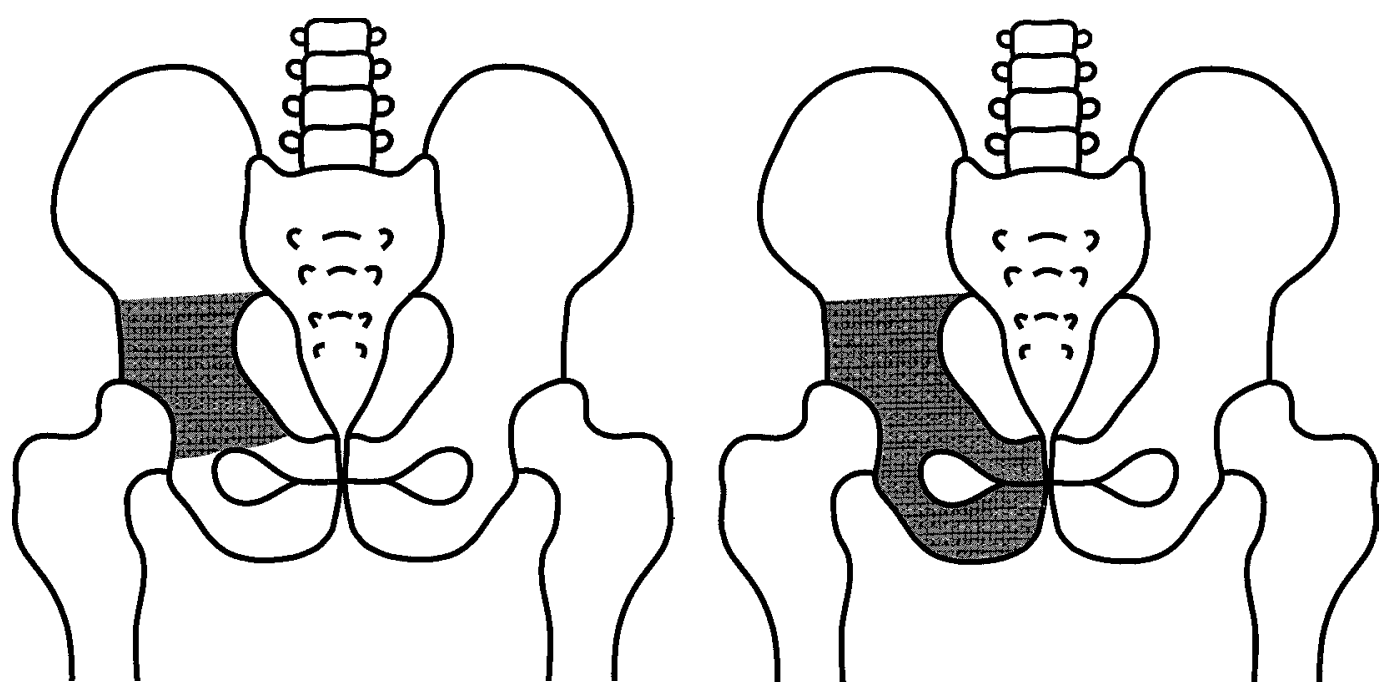

Fig. 1

Diagram of periacetabular excisions for which custom-made pelvic prostheses are used for reconstruction of the defect.

ment Centres. We reviewed all the patients from both centres retrospectively. The indications for excision included chondrosarcoma in 14 patients, histological grade 1 in four and grades 2 or 3 in ten, Ewing's sarcoma in eight, osteosarcoma in four, malignant fibrous histocytoma (MFH) in four, solitary metastatic renal-cell carcinoma in three, plasmacytoma in one and angiosarcoma in one. At diagnosis, staging studies included blood and serum biochemistry, whole body bone scintigraphy, chest radiography, chest CT with plain radiography and CT or MRI of the pelvis. All 35 patients, other than the three with metastatic renal-cell carcinoma, had stage-IIB tumours on the Musculoskeletal Tumor Society grading system. ${ }^{13}$

We obtained clinical data from the hospital databases, case notes and imaging examinations. The average age of the patients was 32 years (13 to 66), the mean follow-up was 49 months (12 to 312 ) and 20 of the 35 patients were female. All the patients with Ewing's sarcoma, osteosarcoma, MFH and angiosarcoma had chemotherapy according to the protocols current at the time. The function of all 21 surviving patients was assessed using the Musculoskeletal Tumor Society system. ${ }^{14}$ No patient was lost to follow-up.

After staging studies, tissue diagnoses were obtained by needle or open biopsy using incisions or approaches along the proposed skin incision for the excision. Clearance biopsies were taken when necessary to confirm absence of tumour at a proposed site of bone transection from the ilium above the tumour. The need for such clearance biopsies has reduced with improved imaging methods, but may still help in confirming the proposed level of transection for the design of the prosthesis and at the operation.

All our patients were catheterised to maintain an empty bladder; some had bowel preparation with an enema. All the operations were performed under general anaesthesia with the patient in the lateral position, and were covered by prophylactic broad-spectrum antibiotics including metronidazole.

All patients had preoperative counselling about the need to eradicate the tumour and were warned that it might be impossible technically to preserve the limb; they all consented to amputation in such conditions.

Technique of operation. The operative details varied from patient to patient, but there were common features. We used part or all of an Ollier and an ilioinguinoperineal approach. ${ }^{15}$ The abdominal muscles were detached from the ilium and the pubis to allow examination of the pelvic cavity and mobilisation of the external iliac vessels, femoral nerve and psoas tendon. The muscles arising from the ilium were detached as far posteriorly as the sciatic notch to allow dislocation of the hip, division of the femoral neck and identification of the resection level in the ilium. Muscle attachments to the pubis and ischium were divided as required. The sciatic nerve and its branches were identified and preserved as were the internal iliac artery and its branches where possible.

The ilium was divided at the predetermined level and the portion of the pelvis containing the intact tumour was removed. Early in the series we routinely used a two-stage procedure (see below) and then developed a one-stage operation. In this custom-made endoprostheses were grouted to the ilium with bone cement and a total hip replacement was completed at the first operation. After replacement the muscles were reattached as far as possible and the wound closed over a suction drain.

After operation, patients rested in bed for an average of two weeks before being allowed to mobilise partially weight-bearing on crutches. At about six weeks, they started intensive physiotherapy to develop the hip and thigh muscles. 


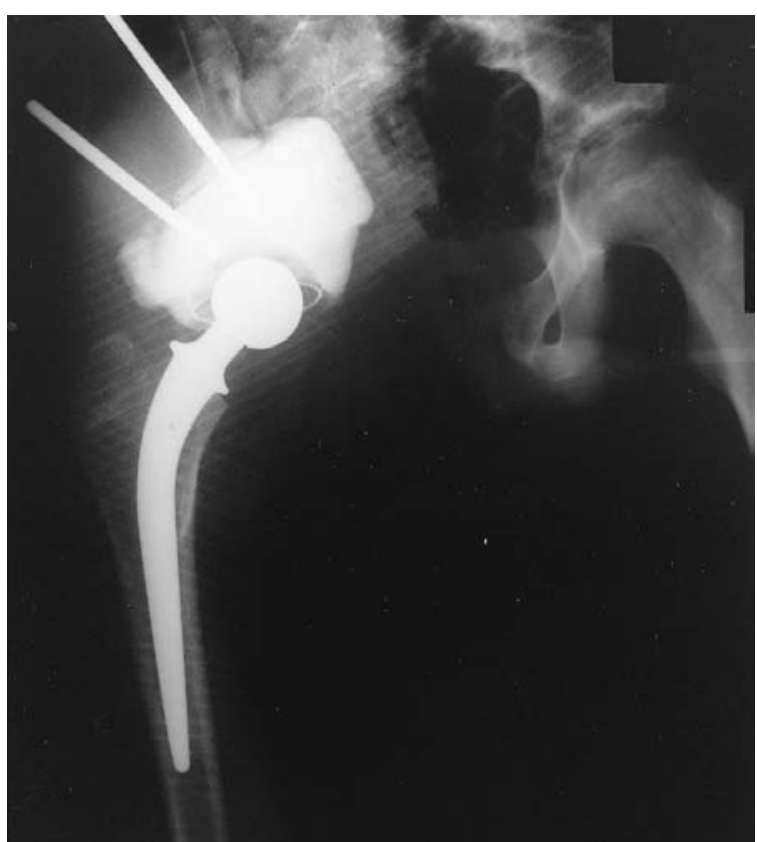

Fig. 2

Radiograph taken after the first stage of a two-stage reconstruction. A temporary acrylic resin spacer and a temporary uncemented femoral prosthesis are shown.

In the whole series 17 patients had two-stage procedures and 18 had a single operation for resection and replacement.

Two-stage procedure. At the first stage, the tumour was excised together with the biopsy track and femoral head and a cast was made of the cut iliac surface. A temporary acrylic resin construct and a conventional cemented femoral component were inserted (Fig. 2). The patient was placed in a hip spica cast and mobilised while the definitive prosthesis was manufactured.

The hemipelvic prosthesis was then custom-made from titanium, using the cast of the iliac surface and the resected specimen as templates for size, shape and orientation (Fig. 3). The second operation was usually about six weeks after the resection. The superior surface of the endoprosthesis was made to fit the cut surface of the ilium accurately and enclose it in a rim extension. The acetabular cavity of the prosthesis included two pins long enough to engage the full depth of the remaining ilium anteriorly and posteriorly. The upper surface of the endoprosthesis was grouted to the ilium with acrylic resin, the two pins were passed into the ilium and the acetabular polyethylene cup was grouted into the acetabular cavity with acrylic resin, which also served to lock the pins in position.

One-stage procedure. We now have the advantage of three-dimensional reconstructions of the pelvis from radiographs and CT scans. This allows the level of transection of the ilium to be determined before the operation, and prostheses can be manufactured before the resection. We use conventional Stanmore femoral prostheses with $32 \mathrm{~mm}$ heads as the femoral replacements.

\section{RESULTS}

At the time of review, 15 patients (43\%) were alive and free from local or metastatic disease at a mean follow-up of 84 months (12 to 312 ). Six (17\%) were alive with metastatic disease at a mean follow-up of 26 months (22 to 32). Twelve $(34 \%)$ had died from metastatic disease and two

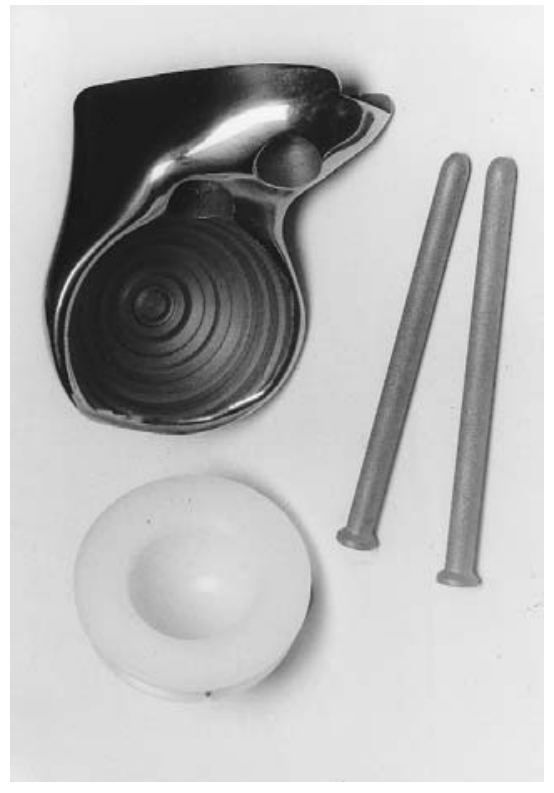

Fig. 3a

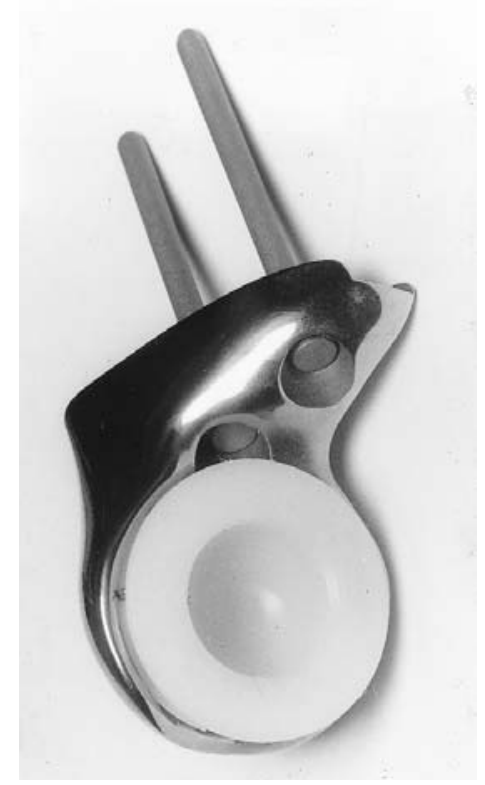

Fig. 3b
Components of a typical prosthesis (a) are also shown in the assembled position (b). 
Table I. Complications in 21 of 35 patients having prosthetic replacement after resections for tumours of the hemipelvis

\begin{tabular}{lll}
\hline & Number & $\begin{array}{l}\text { Percentage of } \\
\text { whole series }\end{array}$ \\
\hline Infection & 9 & 26 \\
Dislocation & 6 & 17 \\
Iatrogenic visceral injury & 2 & 6 \\
Arterial thrombosis & 1 & 3 \\
Pulmonary embolism & 1 & 3 \\
Incisional hernia & 1 & 3 \\
Femoral loosening & 1 & 3 \\
Polyethylene wear & 1 & 3 \\
Secondary haemorrhage & 1 & 3 \\
Sacroiliac pain & 1 & 3 \\
Sciatic nerve palsy caused by tilt of & 1 & 3 \\
pelvic prosthesis &
\end{tabular}

(6\%) from other causes. There had been local recurrence in eight patients $(23 \%)$, three of whom had a later hemipelvectomy to provide local control.

Complications. There were important surgical complications in 21 patients $(60 \%)$. Table I gives the details.

Infection. Deep infection was the commonest complication, seen in nine patients $(26 \%)$. This was due to coagulasenegative staphylococcus in five, Bacterioides fragilis in two, Enterococcus faecalis in one and coliforms in one. In three patients it was possible to control the infection and salvage the prosthesis by surgical debridement and antibiotics and one had a successful two-stage revision. Three patients required excision arthroplasty to eliminate infection and two secondary hindquarter amputation.

Dislocation. Six patients had dislocations (17\%); all were recurrent ( 2 to 5 times, mean 3 ) in the first year and were related to poor function of the hip abductors. Treatment after reduction was by physiotherapy and hydrotherapy; this was successful but one patient required replacement of a worn polyethylene acetabular cup.

Visceral injury. Two patients had injury to the urinary bladder, causing leakage of urine which was confirmed by contrast urography. Conservative management by catheterisation for two to three weeks and antibiotics was successful.

Thromboembolism. One patient had a thrombosis of the femoral artery leading to ischaemia and requiring hemipelvectomy. One patient had a pulmonary embolism, successfully treated by anticoagulation. We did not use routine prophylactic anticoagulation.

Loosening. Two patients developed subsidence and varus tilt of the pelvic endoprosthesis. The oldest and the heaviest patient in the series showed this at six years and pressure on the sciatic nerve caused a foot drop. The patient declined further treatment and continues to be active and mobile with the aid of one walking stick. The other patient, the first with this type of reconstruction, done 26 years ago, showed uncomplicated subsidence and tilt at ten years, but she continues to be active and mobile as a farmer's wife (Fig. 4). Neither patient shows progression of the sub- sidence or tilt at four and 16 years after they were first observed. One young patient had aseptic loosening of the femoral prosthesis, requiring revision at five years.

Back pain. The incidence of significant back pain was low, with one case of sacroiliac pain, for which fusion of the sacroiliac joint has been advised.

Incisional hernia. One patient had an incisional hernia in the inguinal region after resection, which was successfully repaired using polyethylene mesh.

Further operations. A total of 14 patients (40\%) required one or more additional procedures, for infection, gangrene, wear of the acetabular component, local recurrence, secondary haemorrhage, dislocation or loosening of the femoral component.

Function. We assessed function in the 13 patients with pelvic endoprostheses who were free from local or bony metastatic disease. We used the Musculoskeletal Tumor Society system which assesses pain, functional limitation, walking distance, use of support, emotional acceptance and gait. Each of these six variables was assessed on a fivepoint scale, giving a maximum score of 30 points, which we recorded as $100 \%$. The average score was 70\% (50 to 90) (Table II).

Our numbers are small and we found no difference between patients treated by one-stage or by two-stage procedures.

\section{DISCUSSION}

Limb preservation after the resection of malignant periacetabular tumours is technically difficult with many surgical complications despite advances in anaesthesia and imaging, and the improved surgical expertise provided by supraregional treatment centres for bone tumours.

Our review of all such patients treated at the two main treatment centres for bone tumours in the UK shows surgical complications in $60 \%$, with $40 \%$ requiring one or more operations for these. Similar complication rates of about $67 \%$ have been reported by Capanna et al ${ }^{16}$ and Rechl et $\mathrm{al}^{17}$ after the excision of pelvic tumours. Most of the complications result from the surgery involved in the excision of the tumour rather than from the method of reconstruction.

The complication rate after excision of pelvic tumours is marginally greater than that after hindquarter amputation. A previous report from one of our centres by Carter et al ${ }^{18}$ found a complication rate of $41 \%$ after hindquarter amputation with perioperative death in $9 \%$. The types of complication after hindquarter amputations were similar to those after tumour excision and prosthetic reconstruction, including wound infection and necrosis, urinary fistula and infection, tumour embolism, renal failure and myocardial infarction, but there were no perioperative deaths after excision and prosthetic reconstruction.

Function after reconstruction by custom-made hemipelvic prostheses was satisfactory in most patients, reaching 


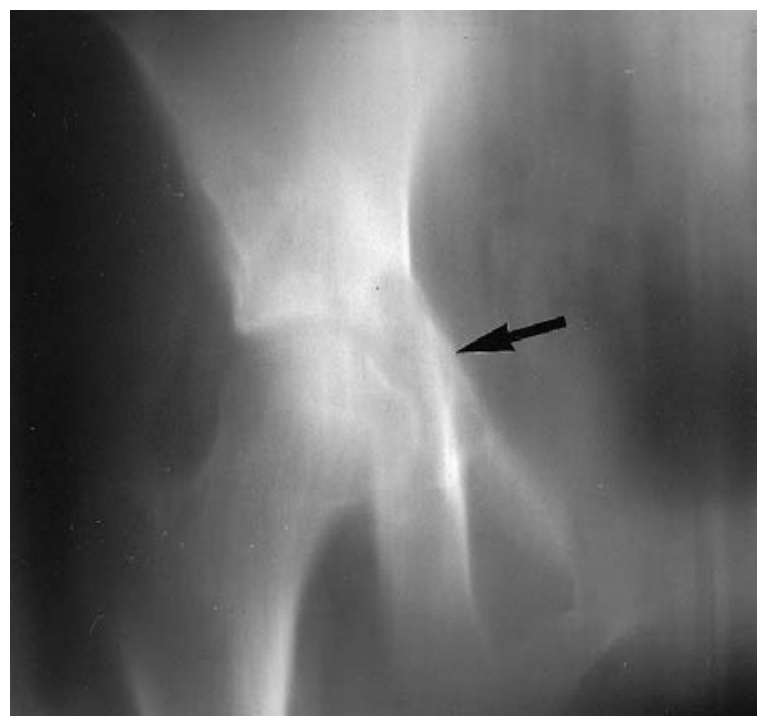

Fig. 4a

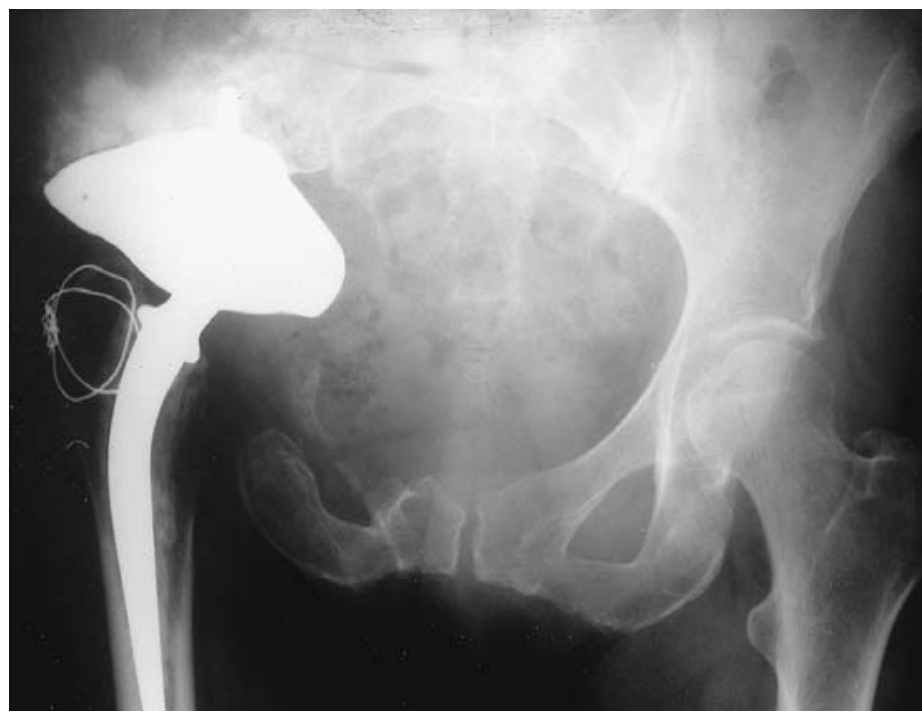

Radiographs of our first patient on whom prosthetic replacement of the hemipelvis was performed 26 years ago.

Figure $4 \mathrm{a}-\mathrm{A}$ tomograph showing a grade-I chondrosarcoma of the acetabulum (arrow). Figure $4 \mathrm{~b}-$ After two-stage excision and prosthetic reconstruction. Figure $4 \mathrm{c}-$ Subsidence and varus tilt of the pelvic prosthesis occurred ten years after reconstruction, but did not progress. The patient is active and mobile, using one walking stick only for long outdoor walks.

Fig. 4c

Table II. Functional scores ${ }^{14}$ for 13 surviving patients with no local recurrence

\begin{tabular}{|c|c|c|c|c|c|c|c|c|c|}
\hline & & Pain & $\begin{array}{l}\text { Recreational } \\
\text { function }\end{array}$ & $\begin{array}{l}\text { Emotional } \\
\text { acceptance }\end{array}$ & Support & $\begin{array}{l}\text { Walking } \\
\text { ability }\end{array}$ & Gait & Total & \\
\hline Case & Normal & 5 & 5 & 5 & 5 & 5 & 5 & 30 & $\%$ score \\
\hline 1 & & 4 & 3 & 4 & 4 & 3 & 2 & 20 & 66 \\
\hline 2 & & 4 & 3 & 4 & 5 & 4 & 4 & 24 & 80 \\
\hline 3 & & 3 & 3 & 3 & 3 & 3 & 2 & 17 & 57 \\
\hline 4 & & 5 & 4 & 4 & 5 & 4 & 4 & 26 & 87 \\
\hline 5 & & 5 & 5 & 4 & 5 & 4 & 4 & 27 & 90 \\
\hline 6 & & 4 & 3 & 4 & 3 & 3 & 4 & 21 & 70 \\
\hline 7 & & 5 & 4 & 4 & 5 & 3 & 3 & 24 & 80 \\
\hline 8 & & 5 & 3 & 4 & 5 & 4 & 5 & 26 & 87 \\
\hline 9 & & 5 & 3 & 4 & 5 & 4 & 5 & 26 & 87 \\
\hline 10 & & 4 & 2 & 3 & 1 & 2 & 3 & 15 & 50 \\
\hline 11 & & 4 & 3 & 3 & 1 & 4 & 3 & 18 & 60 \\
\hline 12 & & 3 & 2 & 2 & 2 & 3 & 3 & 15 & 50 \\
\hline 13 & & 3 & 3 & 4 & 4 & 4 & 3 & 21 & 70 \\
\hline Mean & & 4 & 3 & 4 & 4 & 3 & 3 & 21 & 70 \\
\hline
\end{tabular}


an average of $70 \%$ of normal. The main muscle groups around the hip are usually preserved, since the abductors retain their proximal attachment to the remaining ilium, and psoas is usually preserved unless the proximal femur is also replaced. We believe that preservation of the sciatic nerve is essential for adequate function. If removal of the gluteal vessels and nerves is necessary to resect the tumour, then limb preservation is contraindicated because there would be no abductor function.

The most important complications were local recurrence $(23 \%)$ and infection $(26 \%)$. The rate of local recurrence is similar to that in patients treated by hindquarter amputation in one of our centres. ${ }^{18}$ Other authors have also reported little difference in surgical morbidity and local recurrence rate between patients treated by hindquarter amputation or pelvic resection. ${ }^{4}$ Local recurrence can be treated by repeated local excision or by secondary hindquarter amputation. About $50 \%$ of patients who develop deep infection can be treated satisfactorily by debridement and antibiotics or by two-stage revision. We found that $40 \%$ of such infections were due to anaerobic organisms, which emphasises the need to use metronidazole for the initial antibiotic prophylaxis and to treat established infections.

There was recurrent dislocation of the hip in $17 \%$, usually due to poor muscle function. The recommended treatment is closed reduction and intensive physiotherapy. In our series, one dislocation was secondary to wear of the polyethylene component which was controlled by changing the cup. Thrombosis of the femoral artery, presumably secondary to local trauma, has very serious consequences, which emphasises the importance of careful observation of the limb pulses.

When the iliac resection involves the sacroiliac joint, secondary degenerative changes may follow. To avoid this we have used a 'step' osteotomy which allows a more proximal division of the lateral ilium. We have used dual fixation of the pelvic prosthesis to both ilium and pubis but pubic fixation may be inadequate and loosening often develops. ${ }^{19}$ Our experience indicates that it is not necessary to close the pelvic ring.

Two of our patients developed a varus tilt of the pelvic prosthesis at six and ten years, respectively, after reconstruction; one of them had a secondary palsy of the sciatic nerve, but both have remained active and mobile with no pain requiring regular analgesia. No further radiological progression of the tilt has been seen at four and 16 years after it was first noted, and it seems possible that the tilt was due to bone resorption with resultant subsidence. One patient required revision of the femoral prosthesis at five years after surgery for aseptic loosening, but no other patient has shown radiological or symptomatic aseptic loosening of the pelvic prosthesis which has required revision to date. This reflects the surprisingly good anatomical stability of this design of implant.

Local excision of the pelvis and acetabulum with no reconstruction usually gives poor function, with migration of the femoral head, shortening and instability. ${ }^{8}$ Other methods of reconstruction include the use of allografts, saddle prostheses and arthrodeses all of which have advantages and disadvantages.

Arthrodesis usually gives a better functional outcome than a pseudarthrosis but is difficult with bony union in only about half of the cases. ${ }^{7}$ The complications of arthrodesis include limb shortening, abnormal gait, and back pain; only $40 \%$ have a satisfactory functional outcome. ${ }^{7}$ Hemipelvic allografts carry an increased risk of infection, nonunion, fracture and protrusio acetabuli. ${ }^{11,20}$ The use of sterilised autografts is gaining popularity, but there is no evidence that they behave differently from allografts of the same size, although they should provide a better 'fit'. 11

We consider that our method can achieve adequate excision and provide satisfactory reconstruction and function. The first patient in the series which we report continues to be active and mobile; at 26 years' follow-up she achieved a $70 \%$ score for functional capability.

Conclusions. Prosthetic reconstruction of the hemipelvis after tumour excision is worthwhile in carefully selected patients. It is technically demanding; both patients and surgeons must be aware of the high incidence of complications. The multidisciplinary skills and experience required, and the need for careful follow-up and rehabilitation mean that patients who may require such management should be assessed and treated only in a specialist centre for orthopaedic oncology.

No benefits in any form have been received or will be received from a commercial party related directly or indirectly to the subject of this article.

\section{REFERENCES}

1. Price CH, Jeffree GM. Incidence of bone sarcoma in SW England, 1946-74, in relation to age, sex, tumour site and histology. $B r J$ Cancer 1977;36:511-22.

2. Goorin AM, Perez-Atayde A, Gebhardt M, et al. Weekly high dose Methotrexate and Doxorubicin for osteosarcoma: the Dana-Farber Cancer Institute/The Children's Hospital study III. J Clin Oncol 1978; 5:1178-84.

3. Simon MA, Aschiliman MA, Thomas N, Mankin HJ. Limb-salvage treatment versus amputation for osteosarcoma of the distal end of the femur. J Bone Joint Surg [Am] 1986;68-A:1331-7.

4. Shin K-H, Rougraff BT, Simon MA. Oncologic outcome of primary bone sarcoma of the pelvis. Clin Orthop 1994;304:207-17.

5. Enneking WF. Local resection of malignant lesions of the hip and pelvis. J Bone Joint Surg [Am] 1966;48-A:991-1007.

6. Steel HH. Partial or complete resection of the hemipelvis: an alternative to hindquarter amputation for periacetabular chondrosarcoma of the pelvis. J Bone Joint Surg [Am] 1978;60-A:719-30.

7. Enneking WF, Dunham WK. Resection and reconstruction for primary neoplasms involving the innominate bone. J Bone Joint Surg [Am] 1978;60-A:731-46.

8. Tait RJ, Selmer K, Neff JR. Pelvic resection: oncologic and functional outcome. In: Tan SK, ed. Limb salvage: current trends. Proceedings of the International Symposium ISOLS Singapore, 1993: 299-304.

9. Mnaymneh W, Malinin T, Mnaymneh LG, Robinson D. Pelvic allograft: a case report with a follow-up evaluation of 5.5 years. Clin Orthop 1988;255:128-32.

10. Harrington KD. The use of hemipelvic allografts or autoclaved grafts for reconstruction after wide resections of malignant tumors of the pelvis. J Bone Joint Surg [Am] 1992;74-A:331-41. 
11. Fujinami S, Ogihara Y, Shiokawa Y, Sekiguchi S, Okada G. Autoclaved bone grafting reinforced by fibula strut graft for reconstruction of periacetabular defects. In: Tan SK, ed. Limb salvage: current trends. Proceedings of the International Symposium ISOLS Singapore, 1993:431-3.

12. Donati D, Campana R, Caldora $\mathbf{P}$, et al. Internal hemipelvectomy of the acetabular area using different methods of reconstruction. In: Tan SK, ed. Limb salvage: current trends. Proceedings of the International Symposium ISOLS Singapore, 1993:185-8.

13. Enneking WF, Spanier SS, Goodman MA. The surgical staging of musculoskeletal sarcoma. J Bone Joint Surg [Am] 1980;62-A: 1027-30.

14. Enneking WF, Dunham W, Gebhardt MC, Malawar M, Pritchard DJ. A system for the functional evaluation of reconstructive procedures after surgical treatment of tumours of the musculoskeletal system. Clin Orthop 1993;286:241-6.

15. Crenshaw AH. Surgical approaches. In: Crenshaw AH, ed. Operative orthopaedics. Missouri, St Louis: Mosby, 1992:71-85.
16. Capanna R, van Horn JR, Guernelli N, et al. Complications of pelvic resections. Arch Orthop Trauma Surg 1987;106:71-7.

17. Rechl H, Gradinger R, Ascheri R, Hipp E. Complications in pelvic tumour prostheses. In: Brown KLB, ed. Complications of limb salvage: prevention, management and outcome. Proceedings of the 6th International Symposium on Limb Salvage Montreal, 1991:241-5.

18. Carter SR, Eastwood DM, Grimer RJ, Sneath RS. Hindquarter amputation for tumours of the musculoskeletal system. J Bone Joint Surg [Br] 1990;72-B:490-3.

19. Rechl H, Plötz W, Kaddick C, Gradinger R, Hipp E. Pelvic tumour prostheses: clinical results of 3-D planning and biomechanical investigation. International Symposium on Limb Salvage. Florence, Italy, 1995:67.

20. Tomford W, Johnson M, Mankin HJ. Allograft-prosthesis combinations in limb salvage surgery. In: Langlais F, Tomeno B, eds. Limb salvage: major reconstructions in oncologic and nontumoral conditions. 5th International Symposium, St Malo, France, 1989:423-7. 